\title{
Robot assisted radical cystectomy versus open radical cystectomy in bladder cancer (RACE): study protocol of a non- randomized comparative effectiveness study
}

\author{
C. J. Wijburg ${ }^{1 *}$ (D, C. T. J. Michels ${ }^{1}$, J. R. Oddens' ${ }^{2}$, J. P. C. Grutters ${ }^{3}$, J. A. Witjes ${ }^{4}$ and M. M. Rovers ${ }^{3}$
}

\begin{abstract}
Background: Despite the fact that the cost-effectiveness of robot-assisted radical cystectomy (RARC) is not yet proven, and open radical (ORC) cystectomy is recommended as the standard of care in patients with high-risk non-muscle-invasive and muscle-invasive bladder cancer, the use of RARC is still increasing. The objective of the current ongoing comparative effectiveness trial therefore is to study the (cost-)effectiveness of RARC compared to ORC, both in terms of objective (complication rates, oncological outcomes) and patient-reported (health-related quality of life) outcome measures.

Methods: This study is designed as a non-randomized, multicentre comparative effectiveness trial. Centres with an annual caseload of $>20$ radical cystectomies can include patients after informed consent has been given. Centres that perform RARC must have passed the (initial) learning curve of 40 cases. A total of $338(2 \times 169)$ patients will be enrolled from 23 participating centres (12 ORC, 10 RARC and 1 LRC). Follow-up visits will be scheduled at 1, 3,6 and 12 months. During each follow-up visit, clinical data and health-related quality of life questionnaires will be administered. Costs will be studied using a monthly resource usage questionnaire. Impact on complications and quality of life will be calculated as the average difference between the groups with 95\% confidence intervals, adjusted for potential baseline differences by means of propensity score matching.

Discussion: This study aims to contribute to the development of evidence-based guidelines regarding the most cost-effective surgical technique for radical cystectomy.

Trial registration: Nederlands Trial Register/Dutch Trial Registry, trial identifying number: NTR5362. Registered on 14 August 2015. (http://www.trialregister.nl/trialreg/admin/rctview.asp?TC=5362).
\end{abstract}

Keywords: Cost-effectiveness, Robot-assisted, Radical cystectomy, Bladder cancer, Complications, Quality of life

\footnotetext{
* Correspondence: Cwijburg@rijnstate.nl

'Department of Urology, Rijnstate Hospital, Wagnerlaan 55, 6815, AD,

Arnhem, The Netherlands

Full list of author information is available at the end of the article
}

(c) The Author(s). 2018 Open Access This article is distributed under the terms of the Creative Commons Attribution 4.0 International License (http://creativecommons.org/licenses/by/4.0/), which permits unrestricted use, distribution, and reproduction in any medium, provided you give appropriate credit to the original author(s) and the source, provide a link to the Creative Commons license, and indicate if changes were made. The Creative Commons Public Domain Dedication waiver (http://creativecommons.org/publicdomain/zero/1.0/) applies to the data made available in this article, unless otherwise stated. 


\section{Background}

Radical cystectomy with pelvic lymphadenectomy and urinary diversion is the standard of care for both high-risk non-muscle-invasive bladder cancer and muscle-invasive disease [1]. This surgery is complex and associated with considerable postoperative morbidity, including major and minor complications with probabilities ranging from 13 to 67\% [2-6]. Complications may lead to extra costs, prolonged hospital stay, additional interventions, a reduced quality of life, and a longer time before patients may return to normal activities.

Currently, there are two main surgical techniques to perform a radical cystectomy: robot-assisted (RARC) and open surgery (ORC). ORC is the recommended treatment according to European guidelines, but RARC has been suggested to improve the perioperative morbidity without compromising oncological efficacy, and the percentage of hospitals and surgeons performing RARC is increasing steadily [7]. For example, 11 out of 69 (14.4\%) Dutch centres performed RARC in 2014, and in $201624 \%$ of all radical cystectomies were performed robot assisted [8]. In the USA, the use of robot assisted technique has increased from $1 \%$ in 2004 to $13 \%$ in 2010 [9]. High-level evidence of methodologically sound clinical studies that compare both treatments is, however, lacking. Furthermore, direct costs of robotic surgery are expected to be higher than for open surgery, so investment in this technology can only be justified when additional costs are balanced by a significant benefit regarding patient outcomes.

\section{Existing knowledge}

At the time we designed this study (2015), two systematic reviews (both including 105 studies) had been reported. $[2,10]$ Novara et al. reported on perioperative outcomes and complications after RARC, whereas Yuh et al. reported on oncologic and functional outcomes after RARC. Ninety-three of the 105 studies reported perioperative outcomes and complications of RARC; including 23 comparative studies and 70 surgical series. Most of the surgical series were retrospective single centre studies and only three randomized trials were found.

The overall results showed shorter operative time for ORC, whereas blood loss and hospital stay were better with RARC. Moreover, 90 day complication rates of any grade and grade 3 complications were lower for RARC, whereas high-grade complication and mortality rates were similar [2] Analyses showed no significant difference between RARC and ORC in lymph node yield and rates of positive surgical margins [10]. Very limited data were available with respect to functional outcomes and quality of life. According to both authors, the most relevant limitations of their systematic reviews were the quality of the included studies, the small study populations, the retrospective nature of most series, the short follow-up period, and the lack of standardized definitions $[2,10]$. This lack of methodologically sound data precludes a definite conclusion about the effectiveness of RARC versus ORC.

Up to now, four randomised trials [11-14] have been reported and two others are ongoing (RAZOR [15] and iROC (ClinicalTrials.gov identifier: NCT03049410). Shen et al. [16] performed a meta-analysis of these 4 RCT's [11-14] and presented evidence for a benefit of estimated blood loss, time to diet, similar perioperative complications and oncological outcomes, but a longer operative time in RARC. Furthermore, in the largest trial [13] surgeons performing RARC were still in their learning curve, and another trial only included 20 patients per arm and potential surgeon bias (i.e. there was a different surgeon for each technique) could not be precluded [14].

The two ongoing trials, i.e. RAZOR [15] and iROC, comprise multi center trials. RAZOR is a multi-institutional, randomized, non-inferiority, phase III trial, which aims to enrol 320 patients from 15 participating centres in the USA. They will study oncological outcomes, complications and quality of life of RARC compared to ORC. The primary endpoint is progression-free survival after 2 years. A potential limitation of the RAZOR trial might be the RARC learning curve, as surgeons must have performed only more than 10 RARCs in the past year. The learning curve of RARC is, however, estimated to be between 16 and 30 cases [17-19]. iROC is a prospective multicentre randomised controlled trial comparing the outcomes of intracorporeal RARC (iRARC) with ORC, which aims to enroll 320 patients. The primary outcomes (functional recovery and return to normal activities) will be measured 90 days post-surgery.

\section{Need for a comparative-effectiveness trial}

As described above, all trials performed so far have been limited by sample size and design, or by their application of RARC with extra-corporeal reconstruction. We have chosen for a comparative effectiveness study since observed effectiveness will depend on the complex decision-making process of clinicians selecting therapies, their experience with the technique under study, and on the decisions and actions of patients, including whether they accept or prefer a certain surgical technique. The impact of these issues on effectiveness may be difficult or impractical to assess in a traditional $\mathrm{RCT}$, in which treatment assignments are randomized and may therefore lead to a bias due to the expertise of the surgeons in a center.

\section{Objective}

The objective of the current ongoing comparativeeffectiveness trial is to study the effectiveness of RARC as compared to ORC in patients with bladder cancer and an indication for radical cystectomy in terms of complication rates, health related quality of life, and disease free survival. 


\section{Methods}

\section{Design}

This study is designed as a non-randomized, multicentre comparative effectiveness trial. Patient recruitment is currently being conducted at urology outpatient clinics in five academic tertiary referral centres and eighteen referral centres in the Netherlands. A multicentre approach was chosen to obtain the desired power and to assure representativeness of our sample to the target population. Each centre will perform either RARC or ORC. Centres that perform both RARC and ORC can only participate if they do not select a technique based on patient characteristics, other than described in the exclusion criteria. Only high volume centres (> 20 radical cystectomies per year) can participate in the study. To prevent learning curve bias, only centres that have performed at least 40 RARCs can participate.

\section{Characteristics of participants Inclusion criteria}

Patients eligible for the trial must comply with all of the following:

1) Eighteen years or older.

2) Oncological indication for radical cystectomy.

3) Histologically proven primary muscle invasive transitional cell carcinoma or therapy resistant high-risk non muscle-invasive bladder cancer (CIS, refractory $\mathrm{pTa}-1)$.

4) Tumour is considered non metastatic (cT1a-cT4a, No M0) at the time of inclusion. Patients with $\mathrm{N}_{+}$ status may receive neoadjuvant chemotherapy, but can only be included in the study when preoperative scans show N0.

5) Able to fill in Dutch questionnaires.

6) Provide written informed consent.

\section{Exclusion criteria}

When a patient meets one of the following exclusion criteria, they will be excluded from participation:

1) Previous major abdominal surgery (i.e. existing stomata, status after low anterior resection of the rectum or rectal amputation, status after open aortabifemoral graft, status after right hemicolectomy).

2) Morbid obesity (BMI $\geq 40 \mathrm{~kg} / \mathrm{m} 2$ ).

3) Radical cystectomy is performed in combination with a nephrectomy or a partial colon resection.

4) Pregnancy.

\section{Interventions}

Overall, the surgical techniques for ORC or RARC are standardized procedures. Radical cystectomy with pelvic lymphadenectomy with diversion will be performed in all cases. If the formation of a neobladder is oncologically safe and technically feasible, patients can choose between an ileal conduit and a continent urinary diversion (neobladder). For RARC, one centre performs an extracorporeal technique, whereas all other centres perform a total intracorporeal reconstruction.

The peri-operative protocol has been standardized for all participating centres, including a fast track (ERAS) protocol. All centres are asked to follow the RACE-ERAS protocol, based on the current international guidelines and best available evidence. An identical protocol is used for RARC and ORC, to prevent bias due to different perioperative protocols. Table 1 presents the RACE-ERAS protocol used for this study.

\section{Outcomes}

\section{Primary endpoint}

The primary outcome measure comprises 90 days complications, according to the Clavien-Dindo classification [20].

\section{Secondary endpoints}

Secondary outcomes comprise perioperative morbidity and mortality, 30 days and 1 year complications, health-related quality of life, disease free survival, time to return to normal activity, blood loss and transfusion parameters, operating time, hospital and intensive care stay, oncological outcomes, and costs.

\section{Patient reported outcomes}

Health-related quality of life is measured at baseline (pre-operatively) and post-operatively at 1, 3, 6, and 12 months using three quality of life questionnaires: Functional Assessment of Cancer Therapy-Bladder cancer Cystectomy (FACT-Bl-Cys, formerly FACT-VCI), Bladder Cancer Index, and the 5-level version of the EuroQol 5D (EQ-5D-5 L).

The FACT-Bl-Cys questionnaire is a disease and treatment-specific tool for assessing quality of life following radical cystectomy and urinary diversion [21]. It includes 42 questions dealing with general domains (physical, social/ family, emotional and functional well-being) plus 17 additional items covering urinary, sexual, and bowel function. The Bladder Cancer Index is developed for patients who are diagnosed with bladder cancer and covers a broad range of health and quality of life, including three domains, namely urinary, bowel and sexual functioning, containing 14, 10 and 12 items, respectively [22]. The EQ-5D-5 L consists of a descriptive part and a visual analogue scale (VAS) [23]. The descriptive part comprises the following five dimensions: mobility, self-care, usual activities, pain/discomfort, and anxiety/depression. The VAS records the respondent's self-rated health on a vertical scale ranging from 0 (worst imaginable health state) to 100 (best imaginable health state). 
Table 1 ERAS protocol used for RACE study

\begin{tabular}{|c|c|c|}
\hline Item & Standard & Optional \\
\hline 1. Pre-operative: extensive counselling patient & $\checkmark$ & \\
\hline 2. Correction anaemia $(\mathrm{Hb} \geq 6 \mathrm{mmol} / \mathrm{l})$ & $\checkmark$ & \\
\hline 3. Pre-operative consult dietary specialist & & $\checkmark$ \\
\hline 4. Quit smoking & & $\checkmark$ \\
\hline 5. Physical exercise before surgery & & $\checkmark$ \\
\hline 6. Pre-operative enema: $12 \mathrm{~h}$ before surgery (in case of constipation) & & $\checkmark$ \\
\hline 7. Pre-operative: High calorie fluids, until $2 \mathrm{~h}$ before surgery & $\checkmark$ & \\
\hline 8. Pre-operative: solid food until $6 \mathrm{~h}$ before surgery & $\checkmark$ & \\
\hline 9. Pre-operative: clear fluids, until $2 \mathrm{~h}$ before surgery & $\checkmark$ & \\
\hline 10. Epidural analgesia (thoracal) until max $72 \mathrm{~h}$ after surgery & $\checkmark$ & \\
\hline $\begin{array}{l}\text { 11. Antibiotic prophylaxis, one-shot pre-operative } \\
\text { Cefazoline ( } \leq 80 \mathrm{~kg} 1 \mathrm{~g} ;>80 \mathrm{~kg} 2 \mathrm{~g} \text { ) + Metronidazole }(500 \mathrm{mg}) \text {. May be } \\
\text { repeated if the operation time is longer than the half-life }\end{array}$ & $\checkmark$ & \\
\hline $\begin{array}{l}\text { 12. Anaesthetic considerations } \\
\text { Normothermia, Goal directed fluid therapy, normovolemia, short acting } \\
\text { sedatives, minimalize opioids. }\end{array}$ & $\checkmark$ & \\
\hline 13. Post-operative: nasogastric tube removed directly after surgery & $\checkmark$ & \\
\hline 14. Post-operative: start laxantia post-op day 1 & $\checkmark$ & \\
\hline 15. Post-operative: chewing gum & & $\checkmark$ \\
\hline 16. Post-operative: standard anti-emetica & $\checkmark$ & \\
\hline 17. Post-operative: start mobilisation $6 \mathrm{~h}$ after surgery & $\checkmark$ & \\
\hline 18. Post-operative: fluid diet until first stool & $\checkmark$ & \\
\hline $\begin{array}{l}\text { 19. Post-operative: Thrombosis prophylaxis for four weeks with } \\
\text { 'low molecular weight heparin' }=\mathrm{LMWH} \text { (Fraxiparine } 0,6 \mathrm{cC} \text { ) }\end{array}$ & $\checkmark$ & \\
\hline
\end{tabular}

Resource use will be assessed using the RACE healthcare usage questionnaire, which is based on the iMCQ [24] (to assess medical consumption such as hospital visits, medication use and domestic help) and the PCQ (to measure absence from work due to illness) [25]. This questionnaire will be completed at baseline (pre-operatively) and every month afterwards. It records resource use, such as doctor's visits, medication, hospital admissions, and out-of-pocket expenses such as over-the-counter drugs. Additionally, travelling time to the outpatient clinic and related costs are asked for. Where relevant, (missing) entries will be verified by data from the medical records.

\section{Clinical outcomes}

Perioperative and postoperative measures (e.g. blood transfusion rates, intraoperative fluid requirements, operative time, length of hospital stay and analgesic requirement) are prospectively recorded. Operating time is defined as the skin-to-skin operating time in minutes, not including anaesthetic preparations as these might differ between the participating centres. Total operating room occupation time is also measured.

Pathological data is obtained from pathology reports after surgery with particular emphasis on surgical margin status, total number of lymph nodes removed and their involvement with cancer, as well as pathological stage of the tumour. A standardized form will be used to collect all information pertaining to specimen processing and staging by the participating institutions. In addition, since centres are obliged to complete the national urologic database, the Dutch Urological Association can and will provide us with export files for each RACE participant, which will subsequently be imported in the eCRF (electronic Case Report Form).

\section{Participant timeline}

Timeline is depicted in Fig. 1. Treating urologists in the participating centres will recruit patients that fulfil the in- and exclusion criteria. Information is given about the rationale and the design of the RACE study. Potential participants will receive both the information letter and the informed consent form. Patients will have sufficient time to consider participation. If they agree to participate, informed consent will be signed. Once patients signed informed consent, contact information will be transmitted to the coordinating researcher. Patients will be operated in their own centre within 4 to 6 weeks; from the moment they signed informed consent.

Patients are asked to fill in 3 quality of life questionnaires at five different time points: before the 


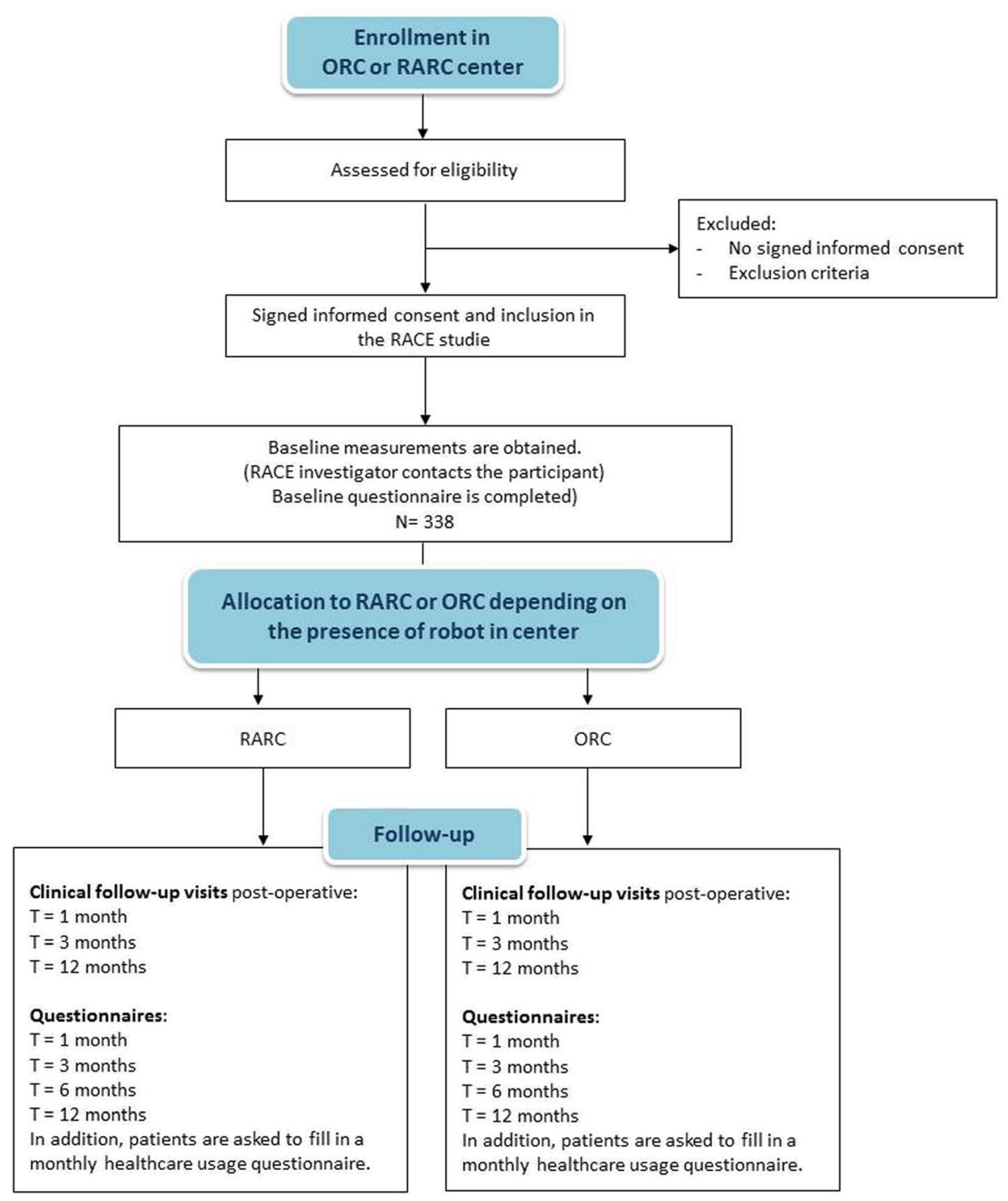

Fig. 1 Flowchart RACE study

operation, and then $1,3,6$ and 12 months after surgery, which will take about ten minutes per questionnaire. In addition, patients are asked to fill in a cost questionnaire monthly, which will take approximately less than five minutes.

Clinical data are registered in a central validated database (eCRF, Research Manager) by (local) researchers. Clinical consultations are scheduled 1, 3, and 12 months after surgery. The data acquisition is presented in Table 2 .

\section{Sample size}

Based on an overall complication percentage of $65 \%$ in the ORC [3] a sample size of $338(2 \times 169)$ patients is required (power 80\%; alpha 5\%) to detect a decrease in the overall complication rate of $15 \%$, i.e. from 65 to $50 \%$.

\section{Recruitment}

Urologists from 23 Dutch centres are involved in patient recruitment. Patient information has been designed in close cooperation with the patient society, i.e. representatives of the Bladder Cancer Patient Society "Leven met Blaas- of Nierkanker" are full members of the RACE project team. Both the support of the Dutch Urology Association and Bladder Cancer Patient Society "Leven met Blaas- of Nierkanker" will help us to include sufficient patients in the trial. Screening and recruitment will continue until the target population $(n=338)$ is achieved.

Data collection methods and data management

Questionnaires will be administered web-based or paper-based, processed and stored digitally using a validated data management system (eCRF, Research Manager). 
Table 2 Standard Protocol Items: Recommendations for Interventional Trials (SPIRIT) flow diagram of study enrolment, interventions and assessments

\begin{tabular}{|c|c|c|c|c|c|c|}
\hline \multirow[b]{3}{*}{ Timeline } & \multicolumn{6}{|l|}{ Study period } \\
\hline & \multirow{2}{*}{$\begin{array}{l}\text { Enrolment } \\
4-6 \text { weeks before Radical Cystectomy }\end{array}$} & \multirow{2}{*}{$\begin{array}{l}\text { Allocation } \\
\text { Radical Cystectomy }\end{array}$} & \multicolumn{3}{|c|}{ Post-allocation } & \multirow{2}{*}{$\begin{array}{l}\text { Close-out } \\
12 \mathrm{~m}\end{array}$} \\
\hline & & & $1 \mathrm{~m}$ & $3 \mathrm{~m}$ & $6 \mathrm{~m}$ & \\
\hline Timepoint & $-t_{1}$ & 0 & $t_{1}$ & $t_{2}$ & $t_{3}$ & $t_{4}$ \\
\hline \multicolumn{7}{|l|}{ Enrolment: } \\
\hline Eligibility screen & $x$ & & & & & \\
\hline Informed consent & $x$ & & & & & \\
\hline Intervention: RARC or ORC & & $x$ & & & & \\
\hline \multicolumn{7}{|l|}{ Assessments: } \\
\hline Quality of Life & $x$ & & $x$ & $x$ & $x$ & $x$ \\
\hline Patient based healthcare usage & $x$ & Monthly & & & & \\
\hline Clinical data & & & $x$ & $x$ & & $x$ \\
\hline
\end{tabular}

Participant application forms (provided by urologists in participating centres), medical history, and clinical data will be registered, processed and stored using eCRF Research Manager. Data will be handled according to the Dutch law (Dutch Data Protection Act). Data will be anonymized by a unique identification number.

\section{Statistical methods \\ Outcomes}

Effects on complications, disease free survival, blood transfusion and organ injury will be calculated as rate differences with $95 \%$ confidence intervals. Effects of surgery on quality of life, pain after surgery, time to return to full activity, operating time and hospital stay will be calculated as mean differences with 95\% confidence intervals. All analyses will be performed on an intention-to-treat basis. To account for potential confounders (including confounding by indication), we will use both multivariate regression models and propensity score methods. Furthermore, a rule-out approach will be used for sensitivity analysis [26] to illustrate how strongly a single unmeasured binary confounder would have to be associated with both the intervention and the end point to fully explain eventual significant findings. Multiple imputation using 10 imputed data sets will be used to handle missing data.

\section{Cost effectiveness analysis}

The economic evaluation will be conducted conform current guidelines [27]. Primary outcome measures for the economic evaluation are societal costs, complications, and quality-adjusted life years (QALYs). To obtain QALYs, the derived health states from the EQ-5D-5 L are converted into utility scores. From these utility scores QALYs will be calculated for each patient using the Area Under the Curve (AUC) method. Resource use will be measured prospectively on a patient level using medical records and patient-completed questionnaires. The friction cost-method will be applied following the Dutch guidelines [27]. Resource use will be multiplied with the corresponding unit costs to obtain total costs for each participating patient. The Dutch guidelines for costing research will be used [27]. For units of care/resources where no guideline or standard price is available actual costs will be determined. Costs of the interventions, RARC and ORC, will be determined through activity-based costing. For RARC, the cost per procedure will depend on the anticipated throughput of patients per year. We will therefore explore a number of scenarios with different throughput.

An incremental cost-effectiveness ratio (ICER), i.e. cost per QALY gained, will be computed. Uncertainty will be determined using the bootstrap method. A cost-effectiveness acceptability curve will be derived to visualize the probability of RARC and ORC being cost-effective, over different thresholds. In addition, the impact of uncertainty surrounding important parameters (for example unit costs) on the ICER will be explored using one-way sensitivity analyses.

While the main purpose of this study involves the comparison between RARC and ORC, a third approach is laparoscopic-assisted cystectomy (LRC). Although this approach is performed in only one Dutch medical centre (i.e. Maxima Medical Centre in Veldhoven), it is interesting to additionally explore the added value of RARC and ORC compared to LRC. During the RACE study, the Maxima Medical Centre will collect the data as described above in the patients that are treated with LRC. Because this involves a small number of patients, decision analytic modelling will be performed to synthesize data from the main RACE study with this additional sample. This will allow us to explore the cost-effectiveness of the three techniques. The model will be constructed in close collaboration with clinical experts, and will comply with the latest guidelines on decision-analytic modelling in health care [28]. 


\section{Data monitoring}

Participation in the RACE-study carries no risks additional to those associated with standard care. Therefore, no Data Monitoring Committee (DMC) is needed. However, the study is conducted in accordance with principles of Good Clinical Practice. For this reason, we have developed a monitoring plan for data collection, aiming for:

1. Full monitoring of informed consents;

2. Monitoring of the first three patients per center. In case of no violation ad random $10 \%$ of patients, to ensure data quality.

3. Source data verification in $10 \%$ of the included patients.

\section{Ethics and dissemination}

\section{Research ethics approval and amendments}

The study protocol, amendments, informed consent form and patient information brochure have been reviewed and approved by the sponsor (ZonMw, The Netherlands Organization for Health Research and Development) and the accredited medical ethics committee (Commission for Research in Human Subjects, in Dutch: Commissie Mensgebonden Onderzoek (CMO), region Arnhem Nijmegen, the Netherlands). Since the enforcement of the Centrale Commissie Mensgebonden Onderzoek (CCMO) External Review Directive in 2012, local medical ethical committees of local participating centers are no longer involved in reviewing the study protocol of multicenter research in the Netherlands and ethical approval obtained, covers all participating centers.

\section{Consent or assent}

Potential participants will be informed by the urologist. Informed consent documents of all participants are obtained and co-signed by clinician who provided the patient information.

\section{Dissemination policy}

Results of the RACE study will be communicated to participants, healthcare professionals and the public through newsletters, publications in peer-reviewed journals and presentations on (inter)national meetings of the Dutch Urology Association and patient society.

\section{Discussion}

Radical cystectomy with pelvic lymphadenectomy and urinary diversion is the standard of care for both high-risk non-muscle-invasive bladder cancer and muscle-invasive disease [1]. Due to improved anaesthesia, better surgical techniques, and centralization of care in high volume centers, reduction in morbidity and mortality has occurred in recent years. Furthermore, the ERAS protocol has also helped to reduce the length of stay and complications [29].
RARC has been suggested to potentially improve the perioperative morbidity. However, high-level evidence of any benefit from RARC over ORC of methodologically sound clinical studies is lacking. This may reflect the complex nature of this procedure as well as the complex decision-making process of clinicians selecting therapies, their experience with the technique under study, and on the decisions and actions of patients, including whether they accept or prefer a certain surgical technique. The impact of these issues on effectiveness may be difficult or impractical to assess in a traditional RCT, in which treatment assignments are randomized and may therefore lead to a bias due to the expertise of the surgeons in a center. So far, four prospective trials have compared RARC with ORC, but each of them has been limited by sample size, design, or their application of RARC with extra-corporeal reconstruction $[11,13,14,30]$.

A comparative effectiveness trial aims to provide high-quality evidence to help patients and clinicians make informed clinical decisions and to assist health systems in improving the quality and cost-effectiveness of clinical care. The major strength of our design is that we compare the preferred treatment strategy (RARC versus ORC) of several centers. Not only the surgeon, but also the team and caseload will influence the outcome for patients. With this comparative effectiveness study we compare outcomes of standardized procedures performed by surgical teams of 23 hospitals (12 ORC, 10 RARC and 1 LRC). The results will provide a valid estimate of the real effectiveness in daily clinical practice. Furthermore, we will be able to study whether the distribution of robotic systems over hospitals and patient allocation to these hospitals is indeed more or less random. Nevertheless, we will also perform propensity score analyses to adjustment for potential confounding (by indication).

Another strength of our study is that only centers that already performed at least 40 RARC-cases and have a caseload of at least 20 cases per year can participate in this study. Several studies have described the learning curve of RARC, which is estimated to be between 16 and 30 cases $[17,19]$. This implies that in our study all centers have passed the learning curve.

At the time of submission of this manuscript, 273 participants have been enrolled in the study. Patient recruitment will continue until September 2018 to achieve the calculated sample size of 338 participants. Due to our experiences and the accrual rate so far, we are confident that we will be able to meet the target sample size and to quantify the effects of RARC as compared to ORC. With the results of this study we aim to contribute to the development of evidence-based guidelines regarding which of the treatment options (RARC or ORC) is the most (cost-)effective. When RARC appears to be cost-effective it might become the standard of care for 
the studied patient population. Patients who met the exclusion criteria (prior extensive abdominal surgery or inability for steep Trendelenburg position) should then be redirected to experienced centers for open surgery. We expect that about $75 \%$ of the cystectomies can be performed with robotic assistance. For RARC to become cost-effective and taking into account a minimal caseload of 45 cases per year due to the learning curve, centralization seems a prerequisite. We will therefore take this centralization question into account in our final analytical decision model and budget impact analysis. If RARC is not cost-effective, ORC should remain the standard treatment, which should be advocated through both international papers, updated guidelines, and various websites presenting our results. For ORC we will also model which centralization option appears to be most promising.

Moreover, we are currently collaborating with researchers from both the RAZOR and iROC trial to enable future data-sharing to perform an individual patient data meta-analysis as to identify relevant subgroups that benefit more or less and to improve the power of studying complication rates and survival.

\section{Trial status}

Recruitment started in March 2016 and is currently ongoing. A total of 273 patients were enrolled by midMarch 2018 and we expect to finish inclusion by the end of 2018. One year followup of last patient and statistical analyses expected by the end of 2019 .

\section{Abbreviations \\ BCl: Bladder Cancer Index; CCMO: Centrale Commissie Mensgebonden Onderzoek; CEA: Cost-Effectiveness Analysis; CMO: Commissie Mensgebonden Onderzoek; EQ-5D-5 L: EuroQol-5 Dimensions-5 Levels; FACT-BI-Cys: Functional Assessment of Cancer Therapy-Bladder cancer Cystectomy; ICER: Incremental cost-effectiveness ratio; iMCQ: Medical Consumption Questionnaire; iPCQ: Productivity Cost Questionnaire; iROC: Trial to compare robotically assisted radical cystectomy with open radical cystectomy; LRC: Laparoscopic-assisted cystectomy; MREC: Medical Research Ethics Committee; NFU: Netherlands Federation of University Medical Centres; NTR: Nederlands Trial Register (Dutch Trial Registry); ORC: Open radical cystectomy; QALY: Quality-adjusted Life Years; RACE: Radical Cystectomy Evaluation; RARC: Robot-assisted radical cystectomy; RAZOR: RAndomiZed Open vs Robotic cystectomy; VAS: Visual analogue scale; WMO: Dutch Medical Research Involving Human Subjects Act}

\section{Acknowledgments}

This trial is non-industry funded. ZonMw is funding the costs of the RACE study, covering budget for personnel and materials, as well as for implementation. The authors would like to thank the following centres that are participating (at least one participant included) in the RACE study at the time of submission of this study protocol:

Amphia, Breda.

Antonius Ziekenhuis, Sneek.

Catharina Ziekenhuis, Eindhoven,

Elisabeth-Twee Steden, Tilburg.

Erasmus Medisch Centrum, Rotterdam.

Franciscus Gasthuis \& Vlietland, Rotterdam.

Gelre Ziekenhuizen, Apeldoorn/Zutphen.

Isala Ziekenhuis, Zwolle.
Jeroen Bosch Ziekenhuis, 's-Hertogenbosch.

Maasstad Ziekenhuis, Rotterdam.

Maxima Medisch Centrum, Veldhoven

Noordwestziekenhuisgroep, Alkmaar.

Radboud Universitair Medisch Centrum, Nijmegen.

Rijnstate Ziekenhuis, Arnhem.

St. Antonius Ziekenhuis, Nieuwegein.

Universitair Medisch Centrum Groningen, Groningen.

Universitair Medisch Centrum Utrecht, Utrecht.

VU Medisch Centrum, Amsterdam.

ZGT, Almelo/Hengelo.

Zuyderland Medisch Centrum, Heerlen.

\section{Funding}

ZonMw (The Netherlands Organisation for Health Research and Development, PO Box 93245, 2509AE The Hague, Netherlands) is funding the RACE study (project number: 843002602). The funding organization had no role in the design, analysis, or presentation of this research. In order to guarantee independence of the RACE study, patients, doctors and centres will not receive financial reimbursement for participating and contributing in the RACE study.

Availability of data and materials

When the RACE study is completed, data will be made available in accordance with ZonMw regulations.

\section{Authors' contributions}

CW, CM, JO, JG, FW and MR all made intellectual contribution to the manuscript, have been involved in drafting the manuscript, have given final approval to the final manuscript and agreed to be accountable.

\section{Ethics approval and consent to participate}

The study protocol, amendments, informed consent form and patient information brochure have been reviewed and approved by the sponsor (ZonMw, The Netherlands Organization for Health Research and Development) and the accredited medical ethics committee (Commission for Research in Human Subjects, in Dutch: Commissie Mensgebonden Onderzoek (CMO), region Arnhem - Nijmegen, the Netherlands). Since the enforcement of the Centrale Commissie Mensgebonden Onderzoek (CCMO) External Review Directive in 2012, local medical ethical committees of local participating centers are no longer involved in reviewing the study protocol of multicenter research in the Netherlands and ethical approval obtained, covers all participating centers. The Medical Research Ethics Committee (MREC) "CMO Regio Arnhem-Nijmegen" determined that the RACE study does not fall under the scope of the Medical Research Involving Human Subjects Act (WMO). There will be no extra burden for the patients participating in this study, as the study does not include additional actions compared to regular care except for the questionnaires.

\section{Consent for publication}

Not applicable.

\section{Competing interests}

Carl Wijburg is a freelance proctor (cystectomy and prostatectomy) with a per day fee from Intuitive Surgical.

Charlotte Michels has no competing interests.

Jorg Oddens has no competing interests.

Janneke Grutters has no competing interests.

Fred Witjes has no competing interests.

Maroeska Rovers has no competing interests.

\section{Publisher's Note}

Springer Nature remains neutral with regard to jurisdictional claims in published maps and institutional affiliations.

\section{Author details}

'Department of Urology, Rijnstate Hospital, Wagnerlaan 55, 6815, AD, Arnhem, The Netherlands. '2Department of Urology, Amsterdam UMC, University of Amsterdam, Meibergdreef 9, 1105 AZ Amsterdam, The Netherlands. ${ }^{3}$ Departments of Operating Rooms and Health Evidence, Radboud university medical center, Geert Grooteplein Noord 21, 6500, HB, Nijmegen, The Netherlands. ${ }^{4}$ Department of Urology, Radboud university medical center, Geert Grooteplein Noord 21, 6500, HB, Nijmegen, The Netherlands. 
Received: 22 May 2018 Accepted: 23 August 2018

Published online: 03 September 2018

\section{References}

1. Alfred Witjes J, Lebret T, Compérat EM, Cowan NC, De Santis M, Bruins HM, Hernández V, Espinós EL, Dunn J, Rouanne M, Neuzillet Y, Veskimäe E, van der Heijden AG, Gakis G, Ribal MJ. Updated 2016 EAU guidelines on muscleinvasive and metastatic bladder Cancer. Eur Urol. 2017;71:462-75.

2. Novara G, Catto JWF, Wilson T, Annerstedt M, Chan K, Murphy DG, Motttrie A, Peabody JO, Skinner EC, Wiklund PN, Guru KA, Yuh B. Systematic review and cumulative analysis of perioperative outcomes and complications after robot-assisted radical cystectomy. Eur Urol. 2015;67:376-401.

3. Musch M, Janowski M, Steves A, Roggenbuck U, Boergers A, Davoudi $Y$, Loewen H, Groeben H, Kroepfl D. Comparison of early postoperative morbidity after robot-assisted and open radical cystectomy: results of a prospective observational study. BJU Int. 2014;113:458-67.

4. Cookson MS, Chang SS, Wells N, Parekh DJ, Smith JA. Complications of radical cystectomy for nonmuscle invasive disease: comparison with muscle invasive disease. J Urol. 2003;169:101-4.

5. Shabsigh A, Korets R, Vora KC, Brooks CM, Cronin AM, Savage C, Raj G, Bochner BH, Dalbagni G, Herr HW, Donat SM. Defining early morbidity of radical cystectomy for patients with bladder cancer using a standardized reporting methodology. Eur Urol. 2009;55:164-74.

6. Konety BR, Allareddy V, Herr H. Complications after radical cystectomy: analysis of population-based data. Urology. 2006;68:58-64.

7. Yu H-Y, Hevelone ND, Lipsitz SR, Kowalczyk KJ, Nguyen PL, Choueiri TK, Kibel AS, Hu JC. comparative analysis of outcomes and costs following open radical cystectomy versus robot-assisted laparoscopic radical cystectomy: results from the US Nationwide inpatient sample. Eur Urol. 2012;61:1239-44.

8. van der Poel H, Stienen J, Wijsman B. Dutch national cystectomy quality registry report. Urograaf. 2016;6:2-5.

9. Leow JJ, Reese SW, Jiang W, Lipsitz SR, Bellmunt J, Trinh Q-D, Chung Bl, Kibel AS, Chang SL. Propensity-matched comparison of morbidity and costs of open and robot-assisted radical cystectomies: a contemporary population-based analysis in the United States. Eur Urol. 2014;66:569-76.

10. Yuh B, Wilson T, Bochner B, Chan K, Palou J, Stenzl A, Montorsi F, Thalmann G, Guru K, Catto JWF, Wiklund PN, Novara G. Systematic review and cumulative analysis of oncologic and functional outcomes after robotassisted radical cystectomy. Eur Urol. 2015;67:402-22.

11. Nix J, Smith A, Kurpad R, Nielsen ME, Wallen EM, Pruthi RS. Prospective randomized controlled trial of robotic versus open radical cystectomy for bladder cancer: perioperative and pathologic results. Eur Urol. 2010; 57:196-201.

12. Parekh DJ, Messer J, Fitzgerald J, Ercole B, Svatek R. Perioperative outcomes and oncologic efficacy from a pilot prospective randomized clinical trial of open versus robotic assisted radical cystectomy. J Urol. 2013;189:474-9.

13. Bochner BH, Dalbagni G, Sjoberg DD, Silberstein J, Keren Paz GE, Donat SM, Coleman JA, Mathew S, Vickers A, Schnorr GC, Feuerstein MA, Rapkin B, Parra RO, Herr HW, Laudone VP. Comparing open radical cystectomy and robot-assisted laparoscopic radical cystectomy: a randomized clinical trial. Eur Urol. 2015;67:1042-50.

14. Khan MS, Gan C, Ahmed K, Ismail AF, Watkins J, Summers JA, Peacock JL, Rimington P, Dasgupta P, Single-centre A. Early phase randomised controlled three-arm trial of open, robotic, and laparoscopic radical cystectomy (CORAL). Eur Urol. 2016;69:613-21.

15. Smith ND, Castle EP, Gonzalgo ML, Svatek RS, Weizer AZ, Montgomery JS, Pruthi RS, Woods ME, Tollefson MK, Konety BR, Shabsigh A, Krupski T, Barocas DA, Dash A, Quek ML, Kibel AS, Parekh DJ. The RAZOR (randomized open vs robotic cystectomy) trial: study design and trial update. BJU Int. 2015;115:198-205.

16. Shen Z, Sun Z. Systematic review and meta-analysis of randomised trials of perioperative outcomes comparing robot-assisted versus open radical cystectomy. BMC Urol. 2016;16:59.

17. Hayn MH, Hussain A, Mansour AM, Andrews PE, Carpentier P, Castle E, Dasgupta P, Rimington P, Thomas R, Khan S, Kibel A, Kim H, Manoharan M, Menon M, Mottrie A, Ornstein D, Peabody J, Pruthi R, Palou Redorta J, Richstone L, Schanne F, Stricker H, Wiklund P, Chandrasekhar R, Wilding GE, Guru KA. The learning curve of robotassisted radical cystectomy: results from the international robotic cystectomy consortium. Eur Urol. 2010;58:197-202.
18. Hayn MH, Hellenthal NJ, Seixas-Mikelus SA, Mansour AM, Stegemann A, Hussain A, Guru KA. Is patient outcome compromised during the initial experience with robot-assisted radical cystectomy? Results of 164 consecutive cases. BJU Int. 2011;108:882-7.

19. Abboudi H, Khan MS, Guru KA, Froghi S, de Win G, Van Poppel H, Dasgupta P, Ahmed K. Learning curves for urological procedures: a systematic review. BJU Int. 2014;114:617-29.

20. Dindo D, Demartines N, Clavien P-A. Classification of surgical complications: a new proposal with evaluation in a cohort of 6336 patients and results of a survey. Ann Surg. 2004;240:205-13.

21. Cookson MS, Dutta SC, Chang SS, Clark T, Smith JA, Wells N. Health related quality of life in patients treated with radical cystectomy and urinary diversion for urothelial carcinoma of the bladder: development and validation of a new disease specific questionnaire. J Urol. 2003;170:1926-30.

22. Gilbert SM, Dunn RL, Hollenbeck BK, Montie JE, Lee CT, Wood DP, Wei JT. Development and validation of the bladder Cancer index: a comprehensive, disease specific measure of health related quality of life in patients with localized bladder cancer. J Urol. 2010;183:1764-9.

23. van Reenen M, Janssen B: EQ-5D-5L User Guide. Basic Information on How to Use the EQ-5D-5L Instrument. Version 2.1, April 2015. 2015.

24. Bouwmans C, Hakkaart van Roijen L, Koopmanschap MA, Krol M, Severens H, Brouwer W: Handleiding iMTA Medical Cost Questionnaire (iMCQ). 2013.

25. Bouwmans C, Krol M, Severens H, Koopmanschap M, Brouwer W, Hakkaartvan Roijen L. The iMTA productivity cost questionnaire: a standardized instrument for measuring and valuing health-related productivity losses. Value Health. 2015;18:753-8.

26. Schneeweiss $\mathrm{S}$. Sensitivity analysis and external adjustment for unmeasured confounders in epidemiologic database studies of therapeutics. Pharmacoepidemiol Drug Saf. 2006;15:291-303.

27. Wyczółkowski M, Juszczak K, Rzepecki M, Drewniak T, Klima W. Studer orthotopic ileal bladder substitute construction - surgical technique and complication management: one-center and 12-year experience. Adv Med Sci. 2010;55:146-52.

28. Caro JJ, Briggs AH, Siebert U, Kuntz KM. ISPOR-SMDM modeling good research practices task force: modeling good research practices--overview: a report of the ISPOR-SMDM modeling good research practices task force--1. Value Health. 2012;15:796-803.

29. Collins JW, Patel H, Adding C, Annerstedt M, Dasgupta P, Khan SM, Artibani W, Gaston R, Piechaud T, Catto JW, Koupparis A, Rowe E, Perry M, Issa R, McGrath J, Kelly J, Schumacher M, Wijburg C, Canda AE, Balbay MD, Decaestecker K, Schwentner C, Stenzl A, Edeling S, Pokupić S, Stockle M, Siemer S, Sanchez-Salas R, Cathelineau X, Weston R, et al. enhanced recovery after robot-assisted radical cystectomy: EAU robotic urology section scientific working group consensus view. Eur Urol. 2016;70:649-60.

30. Messer JC, Punnen S, Fitzgerald J, Svatek R, Parekh DJ. Health-related quality of life from a prospective randomised clinical trial of robot-assisted laparoscopic vs open radical cystectomy. BJU Int. 2014;114:896-902.

\section{Ready to submit your research? Choose BMC and benefit from:}

- fast, convenient online submission

- thorough peer review by experienced researchers in your field

- rapid publication on acceptance

- support for research data, including large and complex data types

- gold Open Access which fosters wider collaboration and increased citations

- maximum visibility for your research: over $100 \mathrm{M}$ website views per year

At BMC, research is always in progress.

Learn more biomedcentral.com/submissions 